synthesis showed that criminalisation and repressive policing disrupted sex workers' safety and risk reduction strategies and access to health services and justice, including where clients are criminalised. Criminalisation and regulatory frameworks exacerbated stigma, racial, economic and other inequalities. In decriminalised contexts, sex workers' relationships with police have improved and they report being better able to refuse clients and insist on condom use.

Conclusions The evidence shows the increased harms associated with sex work criminalisation-including laws and enforcement targeting the sale and purchase of sex, and sex work organisation. These demonstrably harmful sex work policies and laws must be reformed urgently if sex workers' right to health is to be realised.

\section{S20.4 CRIMINALISATION OF HIV TRANSMISSION IN THE ERA OF $\mathrm{U}=\mathrm{U}$}

Michael Brady*. Kings College Hospital, London, UK

10.1136/sextrans-2019-sti.92

Globally, HIV criminalisation continues to exacerbate and perpetuate HIV stigma and discrimination. Over 70 countries have laws that specifically criminalise HIV non-disclosure, exposure or transmission, and 39 countries have used existing criminal laws to prosecute people living with HIV. Society and criminal justice systems have failed to keep up with scientific advances of recent years and, in particular, our understanding of the powerful impact anti-retroviral therapy has on reducing HIV transmission risk. We now know that individuals on effective HIV therapy with an undetectable viral load do not transmit the virus to their sexual partners. This knowledge has not, as yet, translated into any significant change to the application of criminal law. The era of $\mathrm{U}=\mathrm{U}$ (Undetectable = Untransmittable) should support our ability to use scientific evidence to end the criminalisation of HIV and the disproportionate impact this has on marginalised communities and those less able, for whatever reason, to achieve and maintain an undetectable viral load.

Disclosure No significant relationships.

\section{S21 - BUILDING A HUMAN \\ INFRASTRUCTURE FOR STI PREVENTION: BEYOND COMMUNITY ENGAGEMENT, CONSULTATION AND ADVISORY BOARDS}

\section{Wednesday, July 17, 2019 \\ 1:45 PM - 3:15 PM}

\section{S21.1 YOUTH AND COMMUNITY BASED RESEARCH}

Sarah Flicker*. York University, Toronto, Canada

10.1136/sextrans-2019-sti.93

This paper reports on the micro-, meso-, and macro-level impacts of creating and sharing digital stories created by Indigenous youth leaders about HIV prevention activism in
Canada. Eighteen participants created digital stories and hosted screenings in their own communities to foster dialogue. Data for this paper is drawn from individual semi-structured interviews with the youth leaders, audio-recordings of audience reflections, and research team member's field notes collected between 2012-2015 across Canada. Data were coded using NVIVO. A content analysis approach guided analysis. The process of sharing their digital stories had a positive impact on the youth themselves and their communities. Stories also reached policy makers. They challenged conventional public health messaging by situating HIV in the context of Indigenous holistic conceptions of health. The impact(s) of sharing digital stories were felt most strongly by their creators, but rippled out to create waves of change for many touched by them. Participatory visual methodologies can be powerful tools in creating social change and reducing health disparities.

Disclosure No significant relationships.

\section{S21.2 ADDRESSING ANTI-LGBT STIGMA AND MEDICAL MISTRUST THROUGH A DATA-INFORMED APPROACH TO COMMUNITY ENGAGEMENT}

Errol Fields*. Johns Hopkins University School of Medicine, Baltimore, USA

\subsection{6/sextrans-2019-sti.94}

HIV disparities are an ongoing problem affecting LGBT populations, particularly Black gay, bisexual and other men who have sex with men (GBMSM) and transgender women (TGW). The etiology of these disparities is multi-factorial, but medical mistrust and anti-LGBT stigma are key barriers to HIV prevention and care access that likely contribute to disparities. A public health department and university-academic collaboration in partnership with clinical and community partners was funded to improve HIV prevention and care access among Black GBMSM and TGW. Recognizing medical mistrust and anti-LGBT stigma as significant prevention and care access barriers for these communities, the project included the development of Baltimore in Conversation (BIC), a program designed to understand and address these barriers. BIC engaged community voices through small-group conversations about lived experiences as LGBT persons, storytelling nights with presentations of personal narratives of resilience, and photo-story exhibitions of local LGBT persons. Innovative evaluation tools were designed and embedded within BIC. The evaluation sought, through qualitative and quantitative approaches, to inform programming with community perspectives and, ultimately, to determine whether BIC programming reduced medical mistrust and anti-LGBT stigma. The evaluation findings showed evidence of progress towards reducing medical mistrust and anti-LGBT stigma and provided community feedback used to make programming more relevant to experiences and needs of local LGBT communities. For example, analysis of small-group conversation transcripts provided community-informed and locally relevant language and images for an HIV prevention media campaign for Black GBMSM and TGW. Analysis of in-depth interviews of photo-story participants suggested participation led to reductions in internalized stigma and external stigma from important members of their social networks. This public health-academic collaboration created the opportunity to develop, evaluate and improve a public health program through a community-informed 
approach. The overall findings, suggest reductions in medical mistrust and anti-LGBT stigma.

Disclosure No significant relationships.

\section{S21.3 AUTHENTIC AND SUCCESSFUL COMMUNITY ENGAGEMENT FOR STI/HIV PREVENTION, SCREENING AND TREATMENT IN RURAL AND URBAN SETTINGS}

Scott Rhodes*. Wake Forest School of Medicine, Social Science and Health Policy, WinstonSalem, USA

10.1136/sextrans-2019-sti.95

Sexually transmitted infections (STIs) and HIV remain critical public health challenges in the United States. There is a clear need for innovative approaches that identify, prioritize, and address underlying 'upstream' social determinants of health while developing and harnessing community assets to improve STI and HIV prevention, screening, and treatment. Community engagement has emerged as an approach to improve public health outcomes, including reduced STIs and HIV. However, community engagement is difficult, and this presentation provides guidance on the characteristics and strategies of authentic and successful community engagement designed to promote STI and HIV prevention, screening, and treatment in both rural and urban settings. Members of our community partnerships used mixed-methods to identify characteristics of successful community engagement and associated strategies across more than 30 STI and HIV initiatives across the United States. We abstracted data from existing project documentation including proposal documents, project-specific logic models, team and partnership meeting notes, and other materials. Partnership members examined these documents and used an iterative approach with review, discussion, and re-review. A component of our analysis was to identify characteristics and strategies that crossed initiatives, had potential to be generalizable, and could guide future STI and HIV initiatives. Some of the characteristics and strategies that were identified included, staff/team knowledge and unflagging commitment to community engagement; understanding of and commitment to social justice; structural flexibility; strong and charismatic institutional leadership; participation of partners representing diverse multiple sectors; recognizing, acknowledging, and reducing power differentials; shared decision making; embracing and working through conflict; identifying and leveraging talent, strengths, and resources; and using a stepwise approach to build a shared history. Often and incorrectly conflated with advisory boards, qualitative methods, and behavioral and social sciences more broadly, community engagement requires careful forethought, the use of inclusive processes that bring together diverse constituencies, and shared resources and power.

Disclosure No significant relationships.

\section{S21.4 SPURRING INNOVATION IN IMPROVING COMMUNITY ENGAGEMENT FOR STI PREVENTION}

Weiming Tang *. UNC Project-China, Guangzhou, China

10.1136/sextrans-2019-sti.96

Improving the input from the community is an approach for STI Prevention. For example, by engaging the contribution of the community, a 'pay-it-forward' dramatically improved chlamydia and gonorrhea testing among Chinese men who have sex with men (MSM). In this presentation, we summarized how innovative tools were used to engage different groups of the population for STI prevention. First, crowdsourcing approaches were used to solicit the inputs from the community for developing new intervention approaches, designing intervention messages, and evaluating intervention programs. Crowdsourcing involves a group of non-experts and experts working together to solve a problem and then sharing solutions with the public. Second, designathon and hackathon methods were adjusted to develop STI intervention messages and platforms among key populations. For example, in China, a 3-day designathon (hackathon-like event) was hosted to develop an HIV testing service strategy for MSM, while MSM were invited to join the event. In 2019, a hackathon event was also conducted in China to develop a platform for MSM to seek for gay-friendly health service. Third, social-marketing and other participatory approaches were used to develop intervention tools for improving STI services.

Disclosure No significant relationships.

\section{Clinical Case Series}

\section{CCSO1 - CLINICAL CASE SERIES - SYPHILIS: THE GREAT IMITATOR}

\section{Monday, July 15, 2019 7:00 AM - 8:00 AM}

\section{CCSO1.1 NEUROSYPHILIS IS MORE COMMON AMONG MALIGNANT SYPHILIS: BASED ON CASE SERIES EVIDENCE}

Pingyu Zhou* ${ }^{*}$ Lin Zhu. Shanghai Skin Disease Hospital, Shanghai, China

\subsection{6/sextrans-2019-sti.97}

Background Malignant syphilis and neurosyphilis were believed more likely to exist in the HIV positive population. However China's huge and active epidemics of syphilis were much more seen in HIV negative population, thus a considerable amount of the malignant syphilis patients with HIV-uninfected patients might be under the shadow of lower evaluate of neurosyphilis.

Methods This study aimed to investigate the relationship between HIV infection, malignant syphilis and neurosyphilis through a systematic analysis.

Results Clinical characteristics of the malignant syphilis: 26 patients were diagnosed with malignant syphilis and eighteen out of them were HIV negative. They presented different symptoms, and had a more aggressive disease progression with skin rashes. Laboratory findings: 1) A strongly high titre of serum RPR and a sub-acute inflammation of the histopathological finds; The RPR titers of all patients were strongly positive with a range from 1:32 to 1:256. Histopathological examination demonstrated a sub-acute inflammation rich with dermal perivascular plasma cell and neutrophils infiltrate. Numerous spirochetes will be found by immunohistochemistry. 2) WBC, protein and VDRL index in CSF. CSF test revealed the increased WBC count $(>10$ cells $/ \mu \mathrm{L})$ in eleven patients, of which five were with HIV positive. Increased total protein $(>0.5 \mathrm{~g} / \mathrm{L})$ were found in eight patients, and four of them 